\title{
Spontaneous CP violation and Higgs spectra
}

\section{Ulrich Nierste* CERN-TH, CH-1211 Geneva 23}

E-mail: ulrich.nierste@cern.ch

ABstract: A general theorem relating Higgs spectra to spontaneous CP phases is presented. The findings are exemplified with the minimal left-right symmetric model (MLRM), for which an exhaustive analysis of the Higgs sector has been performed. In the limit of a large right-handed scale the MLRM can approach the Standard Model only, if CP-violating phases between the Higgs vacuum expectation values are exactly zero. Otherwise the low-energy model is either a two-Higgs-doublet model or a model with extra $\mathrm{SU}(2)_{\mathrm{L}}$ triplet Higgses.

\section{Introduction}

At present prime experimental effort is devoted to CP violation. In the Standard Model (SM) CP is broken explicitly, through the complex phase in the Cabibbo-KobayashiMaskawa (CKM) matrix [1]. Models with explicit CP violation suffer from a general problem: a CP-noninvariant Lagrangian usually contains more sources of $\mathrm{CP}$ violation than experimentally observed. While the observed CP violation in flavour-changing weak decays complies with the predictions of the SM, the apparent absence of $\mathrm{CP}$ violation in the strong sector remains a mystery. This problem becomes more severe in extensions of the SM; for example the minimal supersymmetric model (MSSM) contains $44 \mathrm{CP}$-violating phases [2] and many of them must be fine-tuned to comply with the observed smallness of CP-violating observables. Models with spontaneous CP violation are therefore an attractive alternative: CP symmetry is imposed on the Lagrangian (so that in particular the Yukawa couplings can be chosen real) and the only sources of CP violation are the complex phases of the vacuum expectation values (VEVs) of Higgs fields. The experimental success of the CKM model suggest that the ball-park of the observed CP violation indeed stems from the CKM phase. In the context of spontaneous CP violation this phase can be generated in two ways: if the spontaneous breakdown of CP occurs at a very high scale well above the electroweak breaking scale $k_{+} \simeq 246 \mathrm{GeV}$, renormalization effects can generate complex Yukawa couplings at low scales. This possibility, however, generically suffers from

\footnotetext{
${ }^{*}$ Speaker.
} 
the same problems as models with explicit $\mathrm{CP}$ violation. The more likely alternative is the second possibility that the complex mass matrices of the quarks are generated from real Yukawa couplings multiplying complex combinations of Higgs VEVs. Then one needs at least two Higgs $\mathrm{SU}(2)_{\mathrm{L}}$ doublets coupled to the quarks [3]. Moreover, both doublets must couple to up and down quarks which implies that there are neutral Higgses with FCNC couplings. In this Talk I consider an arbitrary Higgs sector with two doublet fields $h_{1}$ and $h_{2}$, whose VEVs $\kappa_{1}$ and $\kappa_{2}$ define the electroweak scale $k_{+}=\sqrt{\left|\kappa_{1}\right|^{2}+\left|\kappa_{2}\right|^{2}}$. The Higgs potential $V\left(H_{i}, h_{1}, h_{2}\right)$ shall further contain an arbitrary number of $\mathrm{SU}(2)_{\mathrm{L}}$ singlet fields $H_{i}$ with VEVs $\left|V_{i}\right| \gg k_{+}$. This is the generic situation, if a larger gauge symmetry is broken spontaneously to the electroweak $\mathrm{SM}$ gauge group $\mathrm{SU}(2)_{\mathrm{L}} \times \mathrm{U}(1)_{Y}$ at some high scale $\mathcal{O}\left(\left|V_{i}\right|\right)$. Since a priori $V$ contains couplings between the $H_{i}$ 's and $h_{i}$ 's, there will be mass terms of the form $\lambda_{i j k l} V_{i} V_{j} h_{k} h_{l}$. This is of high phenomenological relevance, since the accurate data from meson-antimeson mixing practically forbid FCNC Higgses with masses of order $k_{+}$. Hence a reasonable model of spontaneous CKM CP violation will aim at masses of order $\left|V_{i}\right|$ for the FCNC Higgses. To this end one must determine the Higgs spectrum in the decoupling limit $\left|V_{i}\right| \rightarrow \infty$ and identify the couplings of the Higgs particles which do not decouple in this limit. The surprising result is that the Higgs spectrum in the decoupling limit is related to the phase $\alpha=\arg \left(\kappa_{1} \kappa_{2}\right)$, which is the source of CKM $\mathrm{CP}$ violation.

In Sect. 2 I will discuss a well-studied model with potential spontaneous CP violation, the minimal left-right symmetric model (MLRM) [4]. The main result is then generalised in Sect. 3. Finally I summarize.

\section{Left-right Symmetry}

The work presented in this section has been obtained in collaboration with G. Barenboim, M. Gorbahn and M. Raidal [5]. The MLRM is ideally suited to study spontaneous CP violation: its Higgs sector is rich enough to allow for spontaneous CP phases and simple enough for an exhaustive study of the Higgs spectrum.

Left-right symmetric models are extensions of the Standard Model (SM) based on the gauge group $\mathrm{SU}(2)_{\mathrm{R}} \times \mathrm{SU}(2)_{\mathrm{L}} \times \mathrm{U}(1)_{\mathrm{B}-\mathrm{L}}$ [4]. The right-handed fermion fields are $\mathrm{SU}(2)_{\mathrm{R}}$ doublets and parity $\mathrm{P}$ is an exact symmetry of the Lagrangian. At a high scale $v_{R}$ well above the electroweak breaking scale $\mathrm{SU}(2)_{\mathrm{R}} \times \mathrm{SU}(2)_{\mathrm{L}} \times \mathrm{U}(1)_{\mathrm{B}-\mathrm{L}} \times \mathrm{P}$ is spontaneously broken to the $\mathrm{SM}$ gauge group $\mathrm{SU}(2)_{\mathrm{L}} \times \mathrm{U}(1)_{\mathrm{Y}}$. The $\mathrm{U}(1)$ charges have a physical interpretation as the difference $\mathrm{B}-\mathrm{L}$ of baryon and lepton number. The hypercharge $\mathrm{Y}$, which is an ad-hoc quantum number of the $\mathrm{SM}$, emerges as the combination $\mathrm{Y}=\mathrm{T}_{3, \mathrm{R}}+(\mathrm{B}-\mathrm{L}) / 2$, where $\mathrm{T}_{3, \mathrm{R}}$ is the third component of the right-handed isospin. The $\mathrm{SU}(2)_{\mathrm{L}} \times \mathrm{SU}(2)_{\mathrm{R}} \times \mathrm{U}(1)_{\mathrm{B}-\mathrm{L}}$ charge assignments for the quark and lepton multiplets are $Q_{L}(1 / 2,0,1 / 3), Q_{R}(0,1 / 2,1 / 3)$, $L_{L}(1 / 2,0,-1)$ and $L_{R}(0,1 / 2,-1)$. The Higgs sector of the MLRM consists of two Higgs triplets $\Delta_{R}, \Delta_{L}$ and a bidoublet $\Phi$ :

$$
\Delta_{L, R}=\left(\begin{array}{cc}
\delta_{L, R}^{+} / \sqrt{2} & \delta_{L, R}^{++} \\
\delta_{L, R}^{0} & -\delta_{L, R}^{+} / \sqrt{2}
\end{array}\right), \quad \Phi=\left(\begin{array}{cc}
\phi_{1}^{0} & \phi_{1}^{+} \\
\phi_{2}^{-} & \phi_{2}^{0}
\end{array}\right) .
$$


The bidoublet transforms under $\mathrm{U}_{L, R} \in \mathrm{SU}(2)_{\mathrm{L}, \mathrm{R}}$ as $\Phi \rightarrow U_{L} \Phi U_{R}^{\dagger}$, i.e. the rows of $\Phi$ are $\mathrm{SU}(2)_{\mathrm{R}}$ doublets and the columns are $\mathrm{SU}(2)_{\mathrm{L}}$ doublets. The triplets transform as $\Delta_{L} \rightarrow U_{L} \Delta_{L} U_{L}^{\dagger}$ and $\Delta_{R} \rightarrow U_{R} \Delta_{R} U_{R}^{\dagger}$. The neutral component of $\Delta_{R}$ acquires a vacuum expectation value (VEV) $v_{R}$, which breaks the $\mathrm{SU}(2)_{\mathrm{R}}$ and $\mathrm{P}$ symmetries. The bidoublet $\Phi$ breaks the electroweak symmetry down to $U(1)_{e m}$ and also breaks CP. One can arrange the VEVs such as $[4,6]$

$$
\left\langle\phi_{1}^{0}\right\rangle=\frac{k_{1}}{\sqrt{2}},\left\langle\phi_{2}^{0}\right\rangle=\frac{k_{2}}{\sqrt{2}} e^{i \alpha},\left\langle\delta_{L}^{0}\right\rangle=\frac{v_{L}}{\sqrt{2}} e^{-i \theta},\left\langle\delta_{R}^{0}\right\rangle=\frac{v_{R}}{\sqrt{2}},
$$

with real and positive $v_{L, R}$ and $k_{1,2}$. The phases $\alpha$ and $\theta$ are CP-violating, $\alpha$ enters the CKM matrix. The most general $\mathrm{C} \times \mathrm{P}$-invariant Higgs potential is [6]

$$
\begin{aligned}
V\left(\Delta_{R},\right. & \left.\Delta_{L}, \Phi\right)=-\mu_{1}^{2} \operatorname{Tr}\left(\phi^{\dagger} \phi\right)-\mu_{2}^{2}\left[\operatorname{Tr}\left(\tilde{\phi} \phi^{\dagger}\right)+\operatorname{Tr}\left(\tilde{\phi}^{\dagger} \phi\right)\right]-\mu_{3}^{2}\left[\operatorname{Tr}\left(\Delta_{L} \Delta_{L}^{\dagger}\right)+\operatorname{Tr}\left(\Delta_{R} \Delta_{R}^{\dagger}\right)\right] \\
& +\lambda_{1}\left[\operatorname{Tr}\left(\phi \phi^{\dagger}\right)\right]^{2}+\lambda_{2}\left\{\left[\operatorname{Tr}\left(\tilde{\phi} \phi^{\dagger}\right)\right]^{2}+\left[\operatorname{Tr}\left(\tilde{\phi}^{\dagger} \phi\right)\right]^{2}\right\}+\lambda_{3}\left[\operatorname{Tr}\left(\tilde{\phi} \phi^{\dagger}\right) \operatorname{Tr}\left(\tilde{\phi}^{\dagger} \phi\right)\right] \\
& +\lambda_{4}\left\{\operatorname{Tr}\left(\phi \phi^{\dagger}\right)\left[\operatorname{Tr}\left(\tilde{\phi} \phi^{\dagger}\right)+\operatorname{Tr}\left(\tilde{\phi}^{\dagger} \phi\right)\right]\right\}+\rho_{1}\left\{\left[\operatorname{Tr}\left(\Delta_{L} \Delta_{L}^{\dagger}\right)\right]^{2}+\left[\operatorname{Tr}\left(\Delta_{R} \Delta_{R}^{\dagger}\right)\right]^{2}\right\} \\
& +\rho_{2}\left[\operatorname{Tr}\left(\Delta_{L} \Delta_{L}\right) \operatorname{Tr}\left(\Delta_{L}^{\dagger} \Delta_{L}^{\dagger}\right)+\operatorname{Tr}\left(\Delta_{R} \Delta_{R}\right) \operatorname{Tr}\left(\Delta_{R}^{\dagger} \Delta_{R}^{\dagger}\right)\right]+\rho_{3}\left[\operatorname{Tr}\left(\Delta_{L} \Delta_{L}^{\dagger}\right) \operatorname{Tr}\left(\Delta_{R} \Delta_{R}^{\dagger}\right)\right] \\
& +\rho_{4}\left[\operatorname{Tr}\left(\Delta_{L} \Delta_{L}\right) \operatorname{Tr}\left(\Delta_{R}^{\dagger} \Delta_{R}^{\dagger}\right)+\operatorname{Tr}\left(\Delta_{L}^{\dagger} \Delta_{L}^{\dagger}\right) \operatorname{Tr}\left(\Delta_{R} \Delta_{R}\right)\right] \\
& +\alpha_{1}\left\{\operatorname{Tr}\left(\phi \phi^{\dagger}\right)\left[\operatorname{Tr}\left(\Delta_{L} \Delta_{L}^{\dagger}\right)+\operatorname{Tr}\left(\Delta_{R} \Delta_{R}^{\dagger}\right)\right]\right\} \\
& +\alpha_{2}\left[\operatorname{Tr}\left(\phi \tilde{\phi}^{\dagger}\right)+\operatorname{Tr}\left(\phi^{\dagger} \tilde{\phi}\right)\right]\left[\operatorname{Tr}\left(\Delta_{R} \Delta_{R}^{\dagger}\right)+\operatorname{Tr}\left(\Delta_{L} \Delta_{L}^{\dagger}\right)\right] \\
& +\alpha_{3}\left[\operatorname{Tr}\left(\phi \phi^{\dagger} \Delta_{L} \Delta_{L}^{\dagger}\right)+\operatorname{Tr}\left(\phi^{\dagger} \phi \Delta_{R} \Delta_{R}^{\dagger}\right)\right]+\beta_{1}\left[\operatorname{Tr}\left(\phi \Delta_{R} \phi^{\dagger} \Delta_{L}^{\dagger}\right)+\operatorname{Tr}\left(\phi^{\dagger} \Delta_{L} \phi \Delta_{R}^{\dagger}\right)\right] \\
& +\beta_{2}\left[\operatorname{Tr}\left(\tilde{\phi} \Delta_{R} \phi^{\dagger} \Delta_{L}^{\dagger}\right)+\operatorname{Tr}\left(\tilde{\phi}^{\dagger} \Delta_{L} \phi \Delta_{R}^{\dagger}\right)\right]+\beta_{3}\left[\operatorname{Tr}\left(\phi \Delta_{R} \tilde{\phi}^{\dagger} \Delta_{L}^{\dagger}\right)+\operatorname{Tr}\left(\phi^{\dagger} \Delta_{L} \tilde{\phi} \Delta_{R}^{\dagger}\right)\right] .(2.3)
\end{aligned}
$$

Here all coefficients are real. Previous studies of the MLRM Higgs sector have used simplifying assumptions on the parameters in (2.3) and/or the VEVs in (2.2). Often these simplifications imply zero CP phases, thereby excluding the case of spontaneous CP violation. In [5] the Higgs mass matrices for arbitrary values of the parameters in (2.3) are computed analytically by expanding in $k_{+}^{2} / v_{R}^{2}$, so that the Higgs spectrum is obtained in the decoupling limit $v_{R} \rightarrow \infty$.

In previous works $[6,7,8]$ it has been argued that sizable CP phases in $(2.2)$ require fine-tuning. In [5] we have found that also scenarios with small or even zero CP phases require fine-tuning, so that this argument cannot be used to disfavour large $\mathrm{CP}$ phases. Fine-tuning is unavoidable, because one wants the ground state of $V$ in (2.3) to produce a gauge hierarchy $v_{R} \gg k_{+}$. Since $V$ is a polynomial, choosing $\mu_{1} \approx \mu_{2} \approx \mu_{3}$ and all couplings of order 1 will result in $k_{+} \approx v_{R} \approx \mu_{i}$. In other words: the desired gauge hierarchy must be encoded into $V$ by choosing a combination of the couplings and the ratios $\mu_{i}^{2} / \mu_{j}^{2}$ of the dimensionful parameters to be of order $k_{+}^{2} / v_{R}^{2}$. Depending on which quantity is chosen $\mathcal{O}\left(k_{+}^{2} / v_{R}^{2}\right)$ different low-energy models emerge. For example, one can find minima of $V$ with $v_{R}=\mathcal{O}\left(\mu_{i}\right)$ and $k_{+}^{2}=\mathcal{O}\left(\alpha_{3} v_{R}^{2}\right)$, that is here the gauge hierarchy 
is defined by he smallness of $\alpha_{3}=\mathcal{O}\left(k_{+}^{2} / v_{R}^{2}\right)$. Since $\alpha_{3}$ appears in the mass matrices of the bidoublet fields one easily verifies that in this example the bidoublet fields do not decouple for $v_{R} \rightarrow \infty$ and the low-energy model is a two-Higgs-doublet model (2HDM) with unacceptable FCNC Higgses [6, 5]. Alternatively one can achieve the desired gauge hierarchy by choosing $2 \rho_{3}-\rho_{1}=\mathcal{O}\left(k_{+}^{2} / v_{R}^{2}\right)$ (along with $\alpha_{3}=\mathcal{O}(1)$ ) and now finds a low-energy model which contains the SM Higgs plus an extra light $\mathrm{SU}(2)_{\mathrm{L}}$ triplet Higgs [5]. This scenario has the attractive feature that one can justify the smallness of $2 \rho_{3}-\rho_{1}$ by any symmetry grouping $\Delta_{L}$ and $\Delta_{R}$ into the same multiplet, such as $\mathrm{SO}(10)$. So in an $\mathrm{SO}(10)$ GUT theory $2 \rho_{3}-\rho_{1}$ will vanish at the GUT scale and become non-zero and small below. Thus here the GUT symmetry explains the gauge hierarchy between $v_{R}$ and $k_{+}$(though not the hierarchy between the GUT scale and $v_{R}$ ). Unfortunately the triplet Higgses in this scenario turn out so small that LEP-I would have discovered them. Most interestingly, the Higgs spectrum in the decoupling limit is related to the CP properties of the vacuum state, i.e. the size of the CP phases $\alpha$ and $\theta$. For illustration I exemplify this relation for the simplistic case with $v_{L}=0$. After decomposing $\phi_{2}^{0}$ from (2.1) as $\phi_{2}^{0}=\left(\phi_{2}^{0 r}+i \phi_{2}^{0 i}+k_{2}\right) \exp (i \alpha) / \sqrt{2}$ the terms relevant for the mass of $\phi_{2}^{0 i}$ are:

$$
\begin{aligned}
\left.\phi_{2}^{0 i} \frac{\partial V}{\partial \phi_{2}^{0 i}}\right|_{\phi_{2}^{0 i}=0}+\left.\frac{\phi_{2}^{0 i 2}}{2} \frac{\partial^{2} V}{\partial \phi_{2}^{0 i 2}}\right|_{\phi_{2}^{0 i}=0}= & \phi_{2}^{0 i} k_{2} \sin \alpha\left[\frac{\alpha_{3}}{2} v_{R}^{2}-2\left(2 \lambda_{2}-\lambda_{3}\right)\left(k_{1}^{2}-k_{2}^{2}\right)\right] \\
& +\phi_{2}^{0 i 2}\left[\frac{\alpha_{3}}{4} v_{R}^{2}+k_{2}^{2} \sin ^{2} \alpha-\left(k_{1}^{2}-k_{2}^{2}\right)\left(2 \lambda_{2}-\lambda_{3}\right)\right] .
\end{aligned}
$$

Since the linear term vanishes in the minimum of the potential, one must have either $\alpha=0$ exactly or the term in square brackets must vanish. In the latter case $\alpha_{3}=\mathcal{O}\left(k_{+}^{2} / v_{R}^{2}\right)$ leading to the $2 \mathrm{HDM}$ in the decoupling limit.

In the general case $\left(v_{L} \neq 0\right) \mathrm{CP}$ phases and Higgs spectra are related as follows:

\begin{tabular}{c|c} 
CP phases & low energy model \\
\hline $\sin \alpha=\mathcal{O}(1)$ & $2 \mathrm{HDM}$ with FCNC Higgses \\
$0<|\sin \alpha| \leq \mathcal{O}\left(k_{+}^{2} / v_{R}^{2}\right)$ & triplet Higgses with $\mathcal{O}\left(v_{L}\right)=\mathcal{O}\left(k_{+}^{2} / v_{R}\right)$ masses \\
$\mathcal{O}\left(k_{+}^{2} / v_{R}^{2}\right) \leq|\sin \alpha| \leq \mathcal{O}\left(k_{+} / v_{R}\right)$ & FCNC and triplet Higgses mix \\
$\alpha=\theta=0$ & Standard Model .
\end{tabular}

In [8] it has been proposed to add an extra pseudo-singlet field to the Higgs potential in order to allow for a large $\alpha$. A qualitative change of our findings for this case occurs, if the VEV $v$ of the new field is large, of order $\mathcal{O}\left(v_{R}\right)$ and has a different phase than $v_{R}$. In this case $\mathrm{CP}$ is broken at the scale $v_{R}$ rather than the electroweak scale. At low energies the model resembles the case with explicit CP violation in $V$. The SM-like spectrum now occurs for $\alpha \neq 0$. If $\mathrm{CP}$ is broken at a high scale, however, a complete analysis of low-energy $\mathrm{CP}$ violation must include leading-log renormalization effects, because $\ln \left(|v| / k_{+}\right)$is large and a tree-level analysis is not accurate.

\section{The General Theorem}

The work presented in this section has been obtained in collaboration with M. Gorbahn [9]. The findings of Sect. 2 on the connection between CP properties and Higgs spectra 
can be generalised to other models with spontaneous $\mathrm{CP}$ violation at the electroweak scale. We find the following theorem:

\section{Decoupling theorem for spontaneous CP violation}

Consider a Higgs potential $V\left(H_{i}, h_{1}, h_{2}\right)$ with $S U(2)_{L}$ doublet fields $h_{1,2}$, whose VEVs $k_{1}$ and $k_{2} e^{i \alpha}$ break the electroweak $S U(2)_{L}$ symmetry, where $\alpha$ is the only source of $\mathrm{CP}$ violation. The $H_{i}$ 's are an arbitrary number of Higgs fields with real VEVs $V_{i} \gg k_{1,2}$. Then either the low energy model is a 2HDM (with flavour-changing Higgs couplings) or the CP phase satisfies $|\sin \alpha| \leq \mathcal{O}\left(k_{1,2}^{2} / V_{i}^{2}\right)$.

\section{Summary}

- If spontaneous $\mathrm{CP}$ violation occurs at the electroweak scale $k_{+}$with minimal Higgs sector (2 doublets coupling to quarks) and these Higgs doublets couple to heavy Higgses with VEVs $V_{i} \gg k_{+}$, then the mass of the FCNC Higgses can only be of order $V_{i}$, if the SCPV phase $\alpha$ decouples as $|\sin \alpha| \leq \mathcal{O}\left(k_{1,2}^{2} / V_{i}^{2}\right)$.

- Analyses within the minimal left-right symmetric model (MLRM) assuming simultaneously $\alpha=\mathcal{O}(1)$ and a SM in the decoupling limit, are incorrect.

- Even for small $\alpha \neq 0$ the low energy MLRM is not the SM, but contains an extra light Higgs triplet.

- In order for the MLRM to approach the SM for $v_{R} \rightarrow \infty$, both CP phases $\alpha$ and $\theta$ must be exactly zero.

\section{References}

[1] M. Kobayashi and T. Maskawa, Prog. Theor. Phys. 49 (1973) 652.

[2] Y. Nir, talk at B physics at the Tevatron, Run-II and beyond, Feb 24-26, 2000, Batavia, USA.

[3] T. D. Lee, Phys. Rev. D 8 (1973) 1226; Phys. Rept. 9 (1974) 143; S. Weinberg, Phys. Rev. Lett. 37 (1976) 657.

[4] J.C. Pati and A. Salam, Phys. Rev. D 10 (1975) 275. R.N. Mohapatra and J.C. Pati, Phys. Rev. D 11 (1975) 566 and 2558. G. Senjanović and R.N. Mohapatra, Phys. Rev. D 12 (1975) 1502. G. Senjanovic, Nucl. Phys. B 153 (1979) 334. R.N. Mohapatra and G. Senjanović, Phys. Rev. D 23 (1981) 165. C. S. Lim and T. Inami, Prog. Theor. Phys. 67 (1982) 1569.

[5] G. Barenboim, M. Gorbahn, U. Nierste and M. Raidal, arXiv:hep-ph/0107121.

[6] N. G. Deshpande, J. F. Gunion, B. Kayser and F. Olness, Phys. Rev. D 44 (1991) 837.

[7] J. Basecq, J. Liu, J. Milutinovic and L. Wolfenstein, Nucl. Phys. B 272 (1986) 145.

[8] G. C. Branco and L. Lavoura, Phys. Lett. B 165 (1985) 327.

[9] M. Gorbahn and U. Nierste, in progress. 